\title{
Aplicación de dos modalidades de riego por goteo en el crecimiento y produccion de camote (Ipomoea batatas L.,variedad INIA 320)
}

Application of two modalities of drip irrigation in the growth and production of sweet potato (Ipomoea batatas L., variety INIA 320)

\author{
Miguel Angel Sanchez Delgado ${ }^{1 *}$; Liz Greca Rivera Serna ${ }^{2}$
}

* Autor de correspondencia

\section{Resumen}

El presente trabajo tuvo por objetivo comparar el rendimiento del cultivo de camote INIA 320, al aplicar el riego por goteo bajo dos modalidades. Se realizó en la parcela demostrativa del Departamento de Recursos Hídricos (DRH) de la Facultad de Ingeniería Agrícola, de la Universidad Nacional Agraria La Molina, entre los meses de febrero a julio del 2015. La parcela experimental tuvo un área de $248 \mathrm{~m}^{2}$ y se trabajó con dos tratamientos: riego por goteo convencional (RC) y riego por goteo intermitente (RI), satisfaciendo una lámina de riego al 100\% de la necesidad hídrica del cultivo. Ambos tratamientos tuvieron la misma cantidad de área y la densidad de plantas. Los resultados obtenidos, se concluye que para ambos tratamientos la cantidad de agua utilizada fue de $3040,45 \mathrm{~m}^{3} \mathrm{ha}^{-1}$ valor que representa aproximadamente un 50\% de la cantidad de agua empleada por los productores que trabajan con riego por gravedad. Asimismo, se analizó los bulbos de humedad, que desarrolla cada tratamiento presentando el riego por goteo intermitente o por ciclos RI una mejor distribución del agua en el suelo el porcentaje de humedad es $6 \%$ mayor respecto al riego por goteo convencional RC. Los rendimientos de la cosecha fueron de $62,75 \mathrm{Tn} \mathrm{ha}^{-1}$ para el riego por goteo intermitente y $57,16 \mathrm{Tn}^{-1} \mathrm{para}^{\mathrm{el}}$ riego por goteo convencional; ambos resultados fueron superiores al rendimiento promedio nacional para el camote INIA 320, que se encuentra alrededor de las $40 \mathrm{Tn}_{\mathrm{ha}}^{-1} \mathrm{y}$ con el riego por gravedad.

Palabras clave: bulbo de humedad; camote; goteo; riego intermitente; rendimiento.

\begin{abstract}
The present work had by objective comparing the yield of the sweet potato INIA 320, applying the drip irrigation under two modalities. The work was carried out in the demonstration plot of the Department of Water Resources (DWR) of the Faculty of Agricultural Engineering of the Universidad Nacional Agraria La Molina, between February and July 2 015. The experimental plot had an area of $248 \mathrm{~m}^{2}$ and two treatments were used: conventional drip irrigation (continuous irrigation) CI and intermittent drip irrigation or IR cycles, satisfying an irrigation sheet at $100 \%$ of the water requirement of the crop. Both treatments had the same amount of area and the amount of plants. According to the results obtained, we concluded that for both treatments the amount of water used was $3040,45 \mathrm{~m}^{3} \mathrm{ha}^{-1}$ value representing approximately $50 \%$ of the amount of water used by producers that work with gravity irrigation. It was also analyzed the humidity bulbs, which develops each treatment with intermittent drip irrigation or by RI cycles. A better distribution of water in the soil, the percentage of humidity is $6 \%$ higher than the conventional drip irrigation treatment CI. The yield of the crop was $62,75 \mathrm{Tn} \mathrm{ha}^{-1}$ for intermittent drip irrigation and 57,16 $\mathrm{Tn} \mathrm{ha}^{-1}$ for conventional drip irrigation, both results were higher than the national average yield for the INIA 320 sweet potato, which Is located around the $40 \mathrm{Tn}^{-1}$ and with the gravity irrigation.
\end{abstract}

Keywords: moisture bulb; sweet potato drip; intermittent irrigation; yield.

\section{Introducción}

En la costa peruana la escasez de agua es uno de los problemas que afecta la producción agrícola, es por ello que se debe racionalizar el uso de los recursos hídricos disponibles. Una forma de racionalizar el recurso hídrico es a través del uso de los sistemas de riego por goteo, es uno de los sistemas que presenta una alta eficiencia en el uso del agua, además de ser un riego localizado, permite la reducción de malezas y a su vez incrementa el rendimiento y calidad del producto. Garcia y Briones (2007) señalan que un sistema de riego por goteo es aquel donde se aplica agua filtrada y el fertilizante dentro o sobre el suelo directamente a cada planta en forma individual. Así mismo, expresan que el agua puede ser suministrada al cultivo con base en una baja tensión de humedad y una alta frecuencia, con lo cual se crea un medio óptimo de humedad necesaria en el suelo. En esta investigación se aplicó el riego intermitente por goteo que consiste en 
suministrar al suelo láminas pequeñas, frecuentes y por ciclos, permitiendo así tener un riego de alta frecuencia, la finalidad de este trabajo fue obtener mayor información de esta nueva forma de aplicar el agua de riego y verificar si resulta o no provechoso su implementación en futuros proyectos agrícolas. Según Morabito y Romay (2001) mencionan que el riego intermitente la aplicación del agua a surcos o melgas regadas por superficie en una serie de períodos de tiempos de apertura y cierre relativamente cortos durante el riego llamados ciclos. Los ciclos tienen dos fases, una de reposición de agua y otra de interrupción, corte o descanso que pueden ser de tiempos iguales o variables, los cuales varían desde cerca de 20 minutos a 2 horas. Este mismo concepto se aplicó en el sistema de riego por goteo. Se utilizó el cultivo de camote como material vegetal por su gran adaptabilidad y por ser un cultivo sencillo trabajar. Según Goyas et al. (1990), su bajo costo y su alto contenido de carbohidratos, proteínas y fibras, hace que esté presente en la alimentación de la población al ser comparado con otros cultivos, provee una mayor cantidad de nutrientes por unidad monetaria, lo que lo hace una alternativa viable para la alimentación de personas de bajos recursos económicos y por la posibilidad de aprovechar su doble propósito de producción de follaje para la alimentación de los animales y las raíces para la alimentación de la personas. En el mundo, cada vez toma mayor fuerza la idea de usar en forma racional y adecuada el recurso hídrico. En la agricultura, donde se usa en grandes cantidades de agua, se producen también grandes pérdidas sobre todo en la utilización de métodos de riego superficial donde el escurrimiento y la percolación profunda son generalmente significativos. Frente a este suceso surge la necesidad de implementar mejoras en el riego, si bien es cierto la tecnificación del riego se viene aplicando en el país, es importante aprovechar el recurso hídrico. Por ello, resulta imprescindible poner en práctica nuevas formas de aplicación del agua de riego que permitan optimizar el uso del agua y lograr obtener mayor productividad y mayor rendimiento de los cultivos.

El objetivo se centró en cuantificar el volumen de agua utilizado por el cultivo de camote variedad INIA-320, al aplicar el riego por goteo convencional y el riego por goteo intermitente. Además de comparar los rendimientos del cultivo de camote por efecto de la aplicación del riego por goteo convencional y el riego por goteo intermitente.

\section{Materiales y métodos \\ Materiales}

El experimento se realizó durante los meses de febrero a julio del 2015, en la parcela demostrativa del departamento de Recursos Hídricos (DRH) de la Facultad de Ingeniería Agrícola, en la UNALM. La parcela experimental cuenta con una instalación para aplicar el sistema de riego presurizado, para esta investigación se tendieron cintas de riego en el campo. Cabe indicar que el área experimental (Figura 1) cuenta con un reservorio de $20 \mathrm{~m}^{3}$, un cabezal de riego y un sistema de fertiriego.

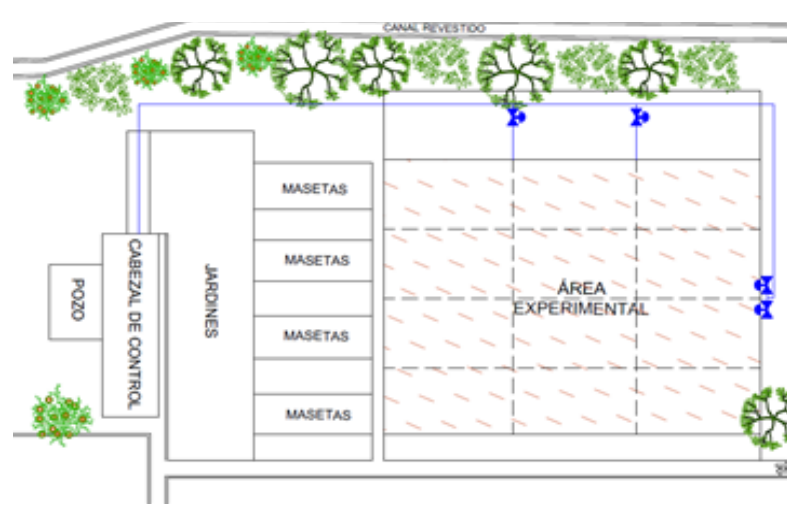

Figura 1. Esquema del área experimental

\section{Material vegetal}

Se utilizaron esquejes de camote de la variedad INIA 320 Amarillo Benjamín. Los esquejes fueron adquiridos del fundo del INIA Donoso, ubicada en Huaral. La semilla fue adquirido de la casa de malla que garantiza que en la cosecha se obtendrá mayor productividad ya que estas se desarrollarán en mejores condiciones que los que crecerán a campo abierto. Además de ello, los esquejes se encontraron libres de patógenos y enfermedades.

\section{Características físicas y químicas del suelo}

La caracterización físico-química se obtuvo al enviar, una muestra representativa de suelo al Laboratorio de Análisis de Agua, Suelo, Medio Ambiente y Fertirriego de la Facultad de Ingeniería Agrícola de la UNALM. La muestra fue obtenida de diferentes puntos del área experimental, a una profundidad de 0 a $40 \mathrm{~cm}$.

Tabla 1. Características físicas del suelo

\begin{tabular}{|c|c|c|c|c|c|}
\hline \multicolumn{4}{|c|}{ Análisis Mecánico } & \multirow{2}{*}{$\mathrm{CE} \mathrm{dSm}{ }^{-1}$} & \multirow{2}{*}{$\mathrm{pH}$} \\
\hline Textura & Arena \% & Limo \% & Arcilla \% & & \\
\hline Franco & 51,12 & 34,72 & 14,16 & 2,99 & 7,73 \\
\hline
\end{tabular}

De acuerdo a lo indicado en la Tabla 1, para un rango de 0 a $40 \mathrm{~cm}$, el suelo presenta una textura franco, este se caracteriza por tener buena capacidad de retención de humedad, buena aireación y adecuada permeabilidad; aptas para el desarrollo del cultivo (Jaramillo, 2002)

\section{Agua}

La fuente de agua proviene del río Rímac a través del canal de Ate y se deriva hacia el área experimental mediante canales revestidos, para ser almacenada en un reservorio de $20 \mathrm{~m}^{3}$. En la Tabla 2 se aprecia las características químicas del agua de riego.

Tabla 2. Características químicas del agua

\begin{tabular}{lcc}
\hline \multicolumn{1}{c}{ Fecha de muestreo } & $\mathrm{pH}$ & $\mathrm{CE}\left(\mathrm{dS} \mathrm{m}{ }^{-1}\right)$ \\
\hline 13 de marzo & 6,87 & 0,75 \\
08 de abril & 6,94 & 0,91 \\
\hline
\end{tabular}




\section{Metodología}

\section{Diseño experimental}

Se utilizó un diseño completamente al azar (DCA), con dos tratamientos y seis repeticiones. El tratamiento testigo (RC) tuvo riego por goteo continuo o convencional y otro bajo el sistema de riego por goteo intermitente (RI), ambos abastecieron la lámina de riego al $100 \%$ de la necesidad hídrica del cultivo. El diseño experimental consideró doce unidades experimentales aplicando dos tratamientos, seis unidades trabajaron con riego por goteo continuo o convencional $(\mathrm{RC})$ y las otras seis con riego por goteo intermitente (RI). cuyo esquema se presenta en la Figura 2 , se observa también que cada unidad experimental ha trabajado de forma independiente, ya que cada unidad ha sido comandada por una mini-válvula que permitió abrir y cerrar el paso de agua. En los tratamientos; cada unidad experimental consta de tres líneas de riego separadas cada $90 \mathrm{~cm}$, los laterales de riego estuvo constituido por cintas de riego, los goteros tuvieron un caudal de 1,6 $1 \mathrm{~h}^{-1} \mathrm{y}$ un distanciamiento de $30 \mathrm{~cm}$.

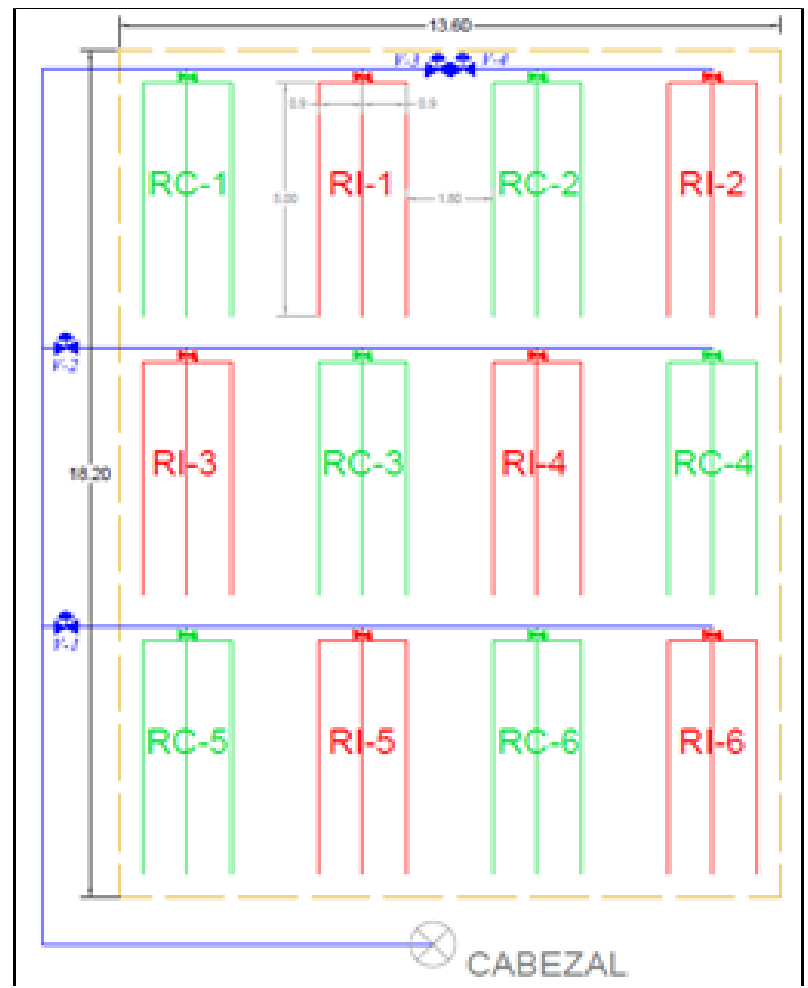

Figura 2. Distribución de parcelas de investigación por tratamiento

\section{Especificaciones de la siembra}

El área sembrada tuvo un total de $156 \mathrm{~m}^{2}$, divididos en 36 surcos con una separación de $0,90 \mathrm{~m}$ y considerando tres surcos por cada unidad experimental. El distanciamiento entre plantas fue de $30 \mathrm{~cm}$, es decir se sembraron 16 esquejes por surco, con un total de 48 esquejes en cada unidad experimental. El período vegetativo fue de 152 días, permitiendo evaluar el nivel de crecimiento y desarrollo del cultivo.

\section{Bulbo de humedad}

En la investigación, se seleccionó los bulbos que tuvieron mejor distribución vertical y horizontal en el suelo.

\section{Elección del ciclo de riego}

Para la elección del ciclo de riego para el tratamiento de riego por goteo intermitente fse realizó pruebas de bulbos de humedad, tal como se observa en las Figuras 3,4 y 5.

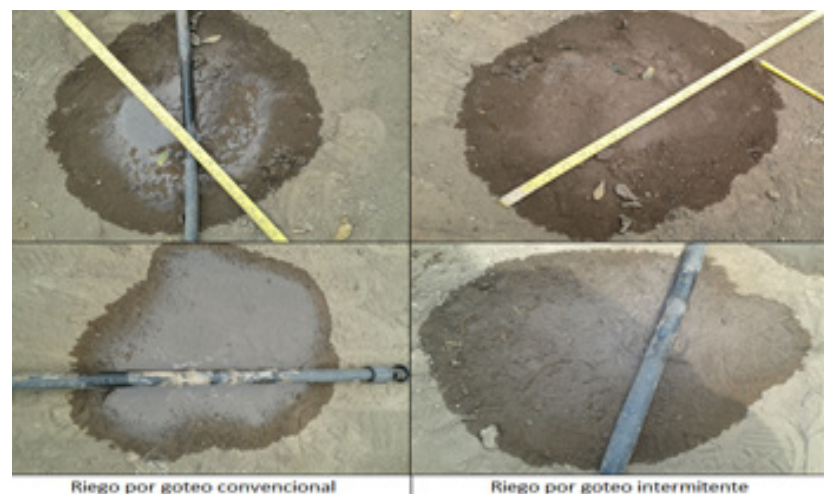

Figura 3. Bulbos de humedad

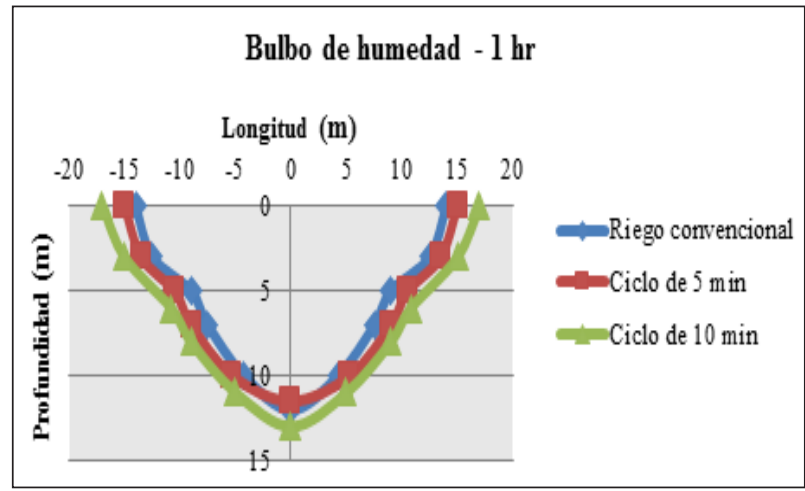

Figura 4. Bulbo de humedad para una hora de riego

De acuerdo a las figuras se puede apreciar una mejor distribución de humedad tanto vertical como horizontal para el ciclo 10-5, que significa 10 minutos de riego continuo y 5 minutos de receso o corte del riego.

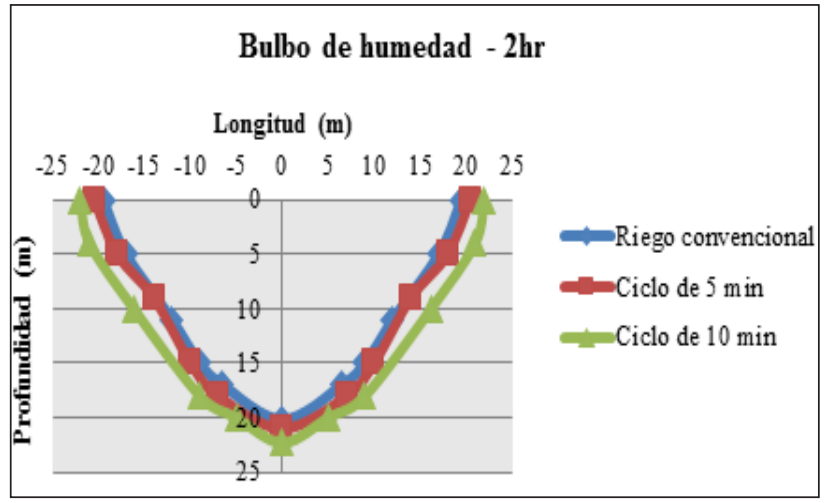

Figura 5. Bulbo de humedad para dos horas de riego 


\section{Resultados y discusión}

\section{Laminas de riego}

Durante toda la etapa fenológica del cultivo, la lámina de riego se calculó de acuerdo a la evapotranspiración diaria. En la fase de establecimiento del cultivo tuvo una duración de 25 días y se aplicaron 10 riegos con una lámina de 110 $\mathrm{mm}$, la segunda etapa tuvo una duración de 30 días y se aplicó 13 riegos con una lámina de $145 \mathrm{~mm}$, la tercera etapa tuvo una duración de 45 días y se aplicaron 18 riegos con una lámina de $190 \mathrm{~mm}$, la última fase tuvo una duración de 52 días y se aplicaron 12 riegos con una lámina de $110 \mathrm{~mm}$.

\section{Cantidad de agua aplicada}

Para la investigación realizada, se consideró aplicar el $100 \%$ de la lámina de agua requerida por la planta, por lo que en ambos tratamientos el volumen de agua y las fechas en que se debía regar es la misma. Para el experimento se consideró que la fase no experimental iba a tener una duración de 20 días, período en que los esquejes debían pegarse al suelo, para ambos tratamientos se aplicó un riego convencional. En la Tabla 4 y 5 se observa el volumen de agua aplicado a cada tratamiento durante la campaña. Como se puede observar, durante la fase no experimental se aplicó menor cantidad de agua que en la fase experimental, la fase experimental corresponde desde el inicio de la tuberización hasta la madurez completa.

Tabla 4. Volumen de agua aplicada por tratamiento $\left(\mathrm{m}^{3}\right.$ ha )

\begin{tabular}{lcrr}
\hline \multicolumn{1}{c}{ Tratamiento } & $\begin{array}{c}\text { Fase no } \\
\text { experimental experimental }\end{array}$ & $\begin{array}{c}\text { Fase } \\
\text { Volumen total } \\
\text { de agua por } \\
\text { campaña }\end{array}$ \\
\hline Riego convencional & 509,75 & 2530,70 & 3040,45 \\
Riego intermitente & 509,75 & 2530,70 & 3040,45 \\
\hline
\end{tabular}

Tabla 5. Volumen de agua aplicada por etapa fenológica del cultivo en $\left(\mathrm{m}^{3} \mathrm{ha}^{-1}\right)$

\begin{tabular}{lrr}
\hline \multicolumn{1}{c}{ Etapa fenológica } & \multicolumn{1}{c}{$\begin{array}{c}\text { Riego } \\
\text { convencional }\end{array}$} & \multicolumn{1}{c}{$\begin{array}{c}\text { Riego } \\
\text { intermitente }\end{array}$} \\
\hline Fase inicial & 615,74 & 615,74 \\
Fase de desarrollo del cultivo & 779,35 & 779,35 \\
Fase de mediados del período & 1041,85 & 1041,85 \\
Fase de finales de período & 603,51 & 603,51 \\
Total de agua aplicada $\left(\mathrm{m}^{3} \mathrm{Ha}^{-1}\right)$ & 3040,45 & 3040,45 \\
\hline
\end{tabular}

La cantidad de agua que se empleó para el experimento fue de $3040,45 \mathrm{~m}^{3} \mathrm{ha}^{-1}$. Este valor representa el 50\% de agua empleada por los productores de camote que usan el riego por gravedad (Chamba, s.f.), por lo que se puede apreciar el ahorro de agua que se tendría al cambiar su sistema a un riego tecnificado, sin dejar de lado otros beneficios (reducción de malezas, mejor uniformidad en el riego, reducción en el empleo de fungicidas, mejor aplicación de fertilizantes por fertiriego, etc). Las cantidades de agua para ambos tratamientos fue la misma, se debe considerar la eficiencia en el uso del agua, ya que por los resultados que se han reportado se ha obtenido que un $10.3 \%$ fue aprovechado por el tratamiento del RI respecto al tratamiento del $\mathrm{RC}$.

\section{Coeficiente de uniformidad}

La uniformidad de riego, en un sistema de riego, generalmente se calcula el coeficiente de uniformidad, por lo que en el presente trabajo de investigación se realizó esta prueba al inicio, a mediados y al final del periodo vegetativo. Los resultados que se muestran en la Tabla 6 se puede decir que el CU a lo largo del experimento ha tenido un resultado favorable ya que se encuentra en el rango propuesto por Pizarro (1996).

Tabla 6. Valores de coeficiente de uniformidad obtenidos en el experimento

\begin{tabular}{lr}
\hline \multicolumn{1}{c}{ Etapa } & \multicolumn{1}{c}{ CU } \\
\hline Inicio & $93,44 \%$ \\
Mediados & $91,90 \%$ \\
Final & $88,70 \%$ \\
\hline
\end{tabular}

\section{Contenido de humedad del suelo}

Para poder estimar la eficiencia en el uso del agua y observar el movimiento del agua en el perfil del suelo por parte de las plantas en tratamiento, se realizaron mediciones del contenido de humedad del suelo antes y después de cada riego realizado. El cultivo de camote tiene la característica de qué forma sus raíces hasta los dos metros de profundidad es por ello que puede tolerar el estrés hídrico. Los riegos han sido realizados dependiendo la pérdida de humedad del suelo, los riegos han sido ligeros y la frecuencia ha sido tal que no se tenga una lámina evaporada acumulada muy elevada.

Es importante indicar que en el experimento se ha evitado tener riegos pesados y exceso de fertilización nitrogenada ya que ocasiona crecimiento de abundante follaje en desmedro de la formación de raíces reservantes. Las fases críticas del camote al déficit hídrico se presentan, después del trasplante y en el momento de llenado de las raíces reservantes. En la etapa de maduración se ha previsto el riego excesivo para evitar la pudrición de las raíces reservantes. A continuación, se muestra la gráfica de comparación de humedad para los dos tratamientos.

En la Figura 6, se muestra el diagrama del contenido de humedad, como se puede apreciar en el tratamiento de riego por goteo intermitente el porcentaje de humedad es ligeramente mayor al del tratamiento de riego por goteo convencional. Durante todo el periodo vegetativo, se trató en lo posible que el porcentaje de humedad se encuentre cerca a la capacidad de campo, la humedad disponible viene a ser $11,89 \%$. Se debe indicar que en los riegos aplicados a los cultivos no se debe permitir un agotamiento mayor del 40 al $60 \%$ de la humedad disponible, a fin de mantener un apropiado nivel de humedad para el cultivo. En nuestro caso la humedad disponible usada por el cultivo ha sido de 3,67\% valor que equivale al $30 \%$ de la humedad disponible. 


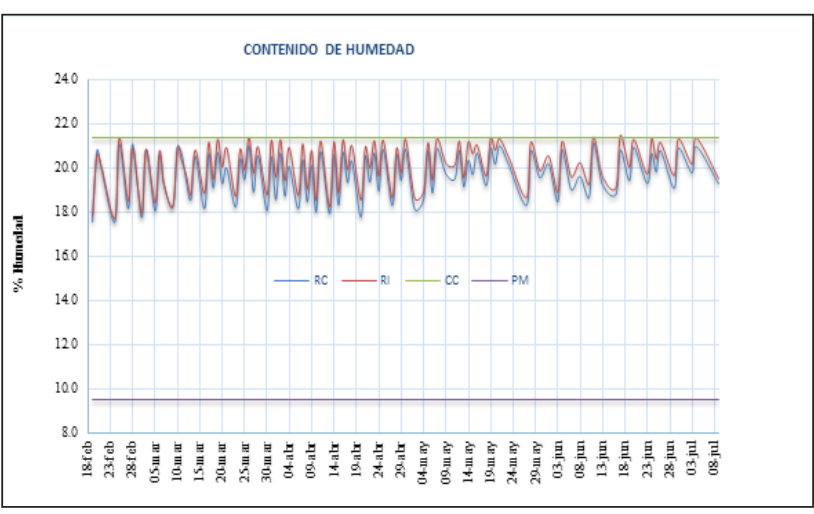

Figura 6. Diagrama del contenido de humedad para ambos tratamientos

\section{Análisis del crecimiento y desarrollo de la planta Área foliar}

El área foliar en el tratamiento del riego por goteo convencional fue menor en todas las etapas fenológicas del cultivo desde que se inició el tratamiento del riego por goteo intermitente. El tratamiento RI tuvo un área foliar máxima a los 77 días después de la siembra obteniendo un $17 \%$ más en comparación con el tratamiento RC.

Para los tratamientos se obtuvo un área foliar máximo a los 77 días después de la siembra, en la curva de crecimiento que se aprecia en la Figura 7 se puede notar que hasta los 42 días el área foliar para ambos tratamientos tienen valores cercanos, a partir de ahí el tratamiento RI presenta mayores valores en comparación con el tratamiento $\mathrm{RC}$, hasta los 112 días en que los valores promedios del área foliar logran tener valores cercanos pero el RI sigue siendo mayor al $\mathrm{RC}$, a partir de ello hasta los 140 días la diferencia entre el tratamiento RI se sigue manteniendo ligeramente mayor respecto al tratamiento $\mathrm{RC}$. Se debe indicar también que en los resultados se ha promediado los valores de las 18 plantas analizadas para cada tratamiento.

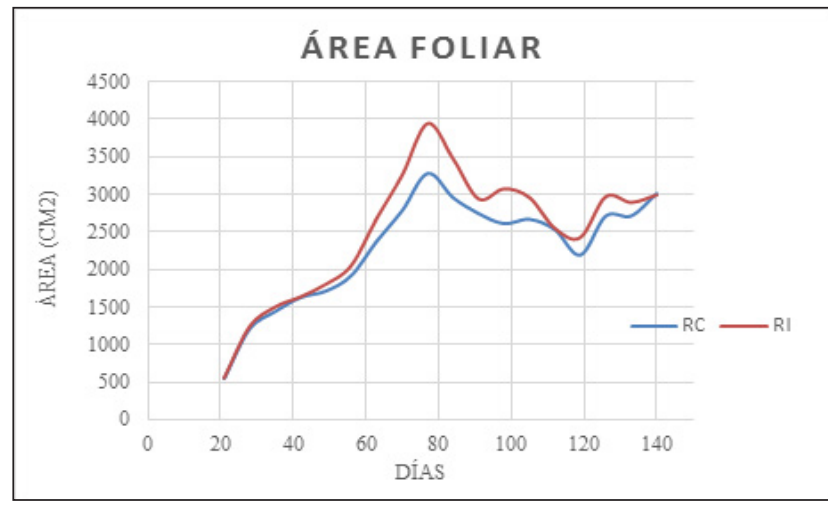

Figura 7. Variación del área foliar según tratamiento

\section{Altura de planta}

A diferencia del área foliar, el tratamiento del riego por goteo convencional tuvo una altura de planta mayor en todas las etapas fenológicas del cultivo en comparación con el tratamiento del riego por goteo intermitente. El tratamiento RC tuvo una altura máxima a los 63 días después de la siembra obteniendo un $2 \%$ más en comparación con el tratamiento RI.

Para ambos tratamientos se tuvo una altura de planta máxima a los 63 días después de la siembra, en la curva de crecimiento que se aprecia en la Figura 8 se puede notar que hasta los 84 días las alturas de planta para ambos tratamientos tienen valores cercanos, a partir de los 84 días el tratamiento $\mathrm{RC}$ se sigue manteniendo ligeramente mayor respecto al tratamiento RI hasta culminar con el período vegetativo del cultivo. Se debe indicar también que en los resultados se han promediado los valores de las 18 plantas analizadas para cada tratamiento.

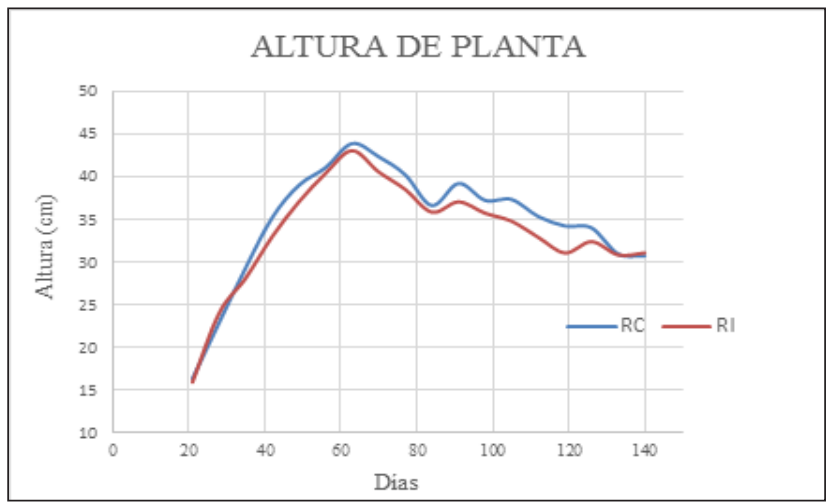

Figura 8. Variación de la altura de planta según tratamiento

\section{Análisis de cosecha}

\section{Rendimiento ( $\mathrm{Tn} \mathrm{ha}^{-1}$ ) total de raíces reservantes}

El rendimiento por tratamiento ha sido: $57,16 \mathrm{Tn}^{-1} \mathrm{~h}^{-1}$ para el tratamiento de riego por goteo convencional y 62,75 Tn $\mathrm{ha}^{-1}$ para el tratamiento de riego por goteo intermitente. El rendimiento para el $\mathrm{RC}$ representa el $91 \%$ respecto a la producción del RI. Los resultados indicados los podemos apreciar en la Figura 9, para el análisis del rendimiento total se ha considerado incluir la prueba de normalidad de errores y la prueba de homogeneidad de varianzas, que son supuestos para poder realizar el análisis de variancia (ANVA). Ambos valores reportan un valor igual a 0,492 que es mayor a 0,05 , por lo que se puede afirmar que se cumplen ambos supuestos para el análisis realizado. El coeficiente de variabilidad en el análisis estadístico del rendimiento total $\left(\mathrm{Tn} \mathrm{ha}^{-1}\right)$ ha salido debajo del $30 \%$ que es un valor aceptado para trabajos realizados en campo. Se debe indicar que el análisis realizado fue considerando un nivel de significancia al 95\% $(\alpha=0,05)$.El análisis de variancia (ANVA) para la variable del rendimiento total nos indica que el rendimiento medio del RC no es diferente al rendimiento medio del RI, por lo que el resultado indica que no existen diferencias significativas entre los dos tratamientos analizados con un coeficiente de variabilidad de $22,65 \%$. 


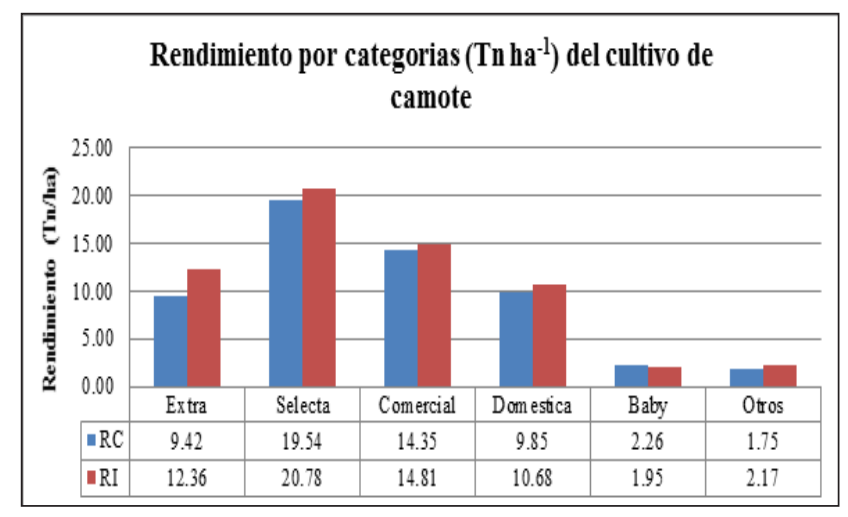

Figura 9. Rendimiento por categorías y tratamiento

Tabla 7. Análisis estadístico para el rendimiento total

\begin{tabular}{ccccc}
\hline Tratamiento & Media $\left(\mathrm{Tn} \mathrm{Ha}^{-1}\right)$ & $\mathrm{N}$ & Tukey & ANVA \\
\hline RI & 62,75 & 6 & $\mathrm{~A}$ & $\mathrm{NS}$ \\
$\mathrm{RC}$ & 57,16 & 6 & $\mathrm{~A}$ & $\mathrm{NS}$ \\
\hline
\end{tabular}

La prueba de comparación múltiple de medias de Tukey, nos confirma los resultados del análisis de variancia ya que se ha trabajado con un par de tratamientos, que nos indica que no existen diferencias significativas entre el tratamiento RC y el RI, ya que ambas comparten la misma letra. Los valores obtenidos en el trabajo han sido favorables ya que se tienen valores más altos al trabajar con riego presurizado. En la variedad amarillo Benjamín el promedio nacional por hectárea esta alrededor de $40 \mathrm{Tn}$ $\mathrm{ha}^{-1}$, sin embargo en la estación experimental Donoso en Huaral se ha logrado conseguir un rendimiento de hasta $50 \mathrm{Tn} \mathrm{ha}^{-1}$, considerando que se trabaja con riego por gravedad.

\section{Rendimiento (Tn $\left.\mathrm{ha}^{-1}\right)$ de raíces reservantes por categoría}

La producción total estuvo compuesta por la clasificación: primera (135 gramos a mas), segunda (37-134 gramos) y descarte (0-36 gramos).

El CIP clasifica el rendimiento del cultivo, en base al peso de cada raíz reservante. Los resultados obtenidos se presentan en la Tabla 8 .

Tabla 8. Clasificación del rendimiento según tratamiento

\begin{tabular}{lrrrr}
\hline \multirow{2}{*}{ Clasificación } & \multicolumn{4}{c}{ Rendimiento } \\
\cline { 2 - 5 } & \multicolumn{2}{c}{$\mathrm{RC}$} & \multicolumn{3}{c}{$\mathrm{RI}$} \\
\hline & \multicolumn{1}{c}{$\mathrm{Tn} \mathrm{ha}^{-1}$} & \multicolumn{1}{c}{ Tnha $^{-1}$} & \multicolumn{1}{c}{$\%$} \\
\hline Primera & 28,96 & 50,67 & 33,14 & 52,82 \\
Segunda & 24,19 & 42,33 & 25,49 & 40,62 \\
Descarte & 4,00 & 7,01 & 4,12 & 6,56 \\
Total & 57,16 & 100,00 & 62,75 & 100,00 \\
\hline
\end{tabular}

En las gráficas se puede notar que el tratamiento RI presenta ligeramente mayores valores en la clasificación de primera calidad (extra y selecta), mientras que para la segunda y tercera calidad los resultados son similares.

De acuerdo a los resultados obtenidos en el análisis estadístico, se puede notar que no existe diferencia significativa entre los tratamientos, los resultados indicaron que en la categoría de primera calidad se presentó un 12,6\% más en el rendimiento del $\mathrm{RI}$ respecto al $\mathrm{RC}$, a diferencia de la categoría de segunda calidad y descarte donde se obtuvieron resultados similares; por lo que el riego por goteo intermitente estaría logrando buenos resultados respecto al riego por goteo convencional. Este resultado es favorable ya que los agricultores vienen aplicando un sistema de riego por surcos y sería importante comparar no solo el rendimiento obtenido sino también la eficiencia del uso del agua que se verá más adelante.

En el análisis del rendimiento de primera y segunda calidad, se ha considerado incluir la prueba de normalidad de errores y la prueba de homogeneidad de varianzas, que son supuestos para poder realizar el análisis de variancia (ANVA). Ambos valores reportan un p-valor mayor a 0,05, por lo que se puede afirmar que se cumplen ambos supuestos en los análisis realizados. El coeficiente de variabilidad, para los análisis realizados han salido debajo del 30\%, salvo en el análisis de rendimiento de primera calidad cuyo valor es cercano al $30 \%$, que es donde el rendimiento para ambos tratamientos considerando las seis repeticiones ha sido la que ha presentado valores más variables comparado con el rendimiento de segunda calidad. Se debe indicar que el análisis realizado para el rendimiento de primera y segunda calidad fue considerando un nivel de significancia al $95 \%(\alpha=0,05)$.

Los resultados del análisis de variancia, el coeficiente de variabilidad y la comparación de medias de Tukey lo podemos apreciar en la Tabla 9, que como resultado nos indica que no existen diferencias significativas entre los tratamientos analizados, con un nivel de significancia al $95 \%(\alpha=0,05)$.

En el análisis del ANVA se obtuvo como resultado que los tratamientos no tuvieron diferencias significativas, al realizar el análisis de comparación múltiple de medias de Tukey, se confirman los resultados del ANVA ya que al tener la misma letra como resultado se puede decir que no existen diferencias significativas entre ambos tratamientos.

Tabla 9. Análisis estadístico para el rendimiento de primera y segunda calidad

\begin{tabular}{|c|c|c|c|c|c|}
\hline Categoría & Tratamiento & Media(Tn ha-1) & Tukey & ANVA & $\mathrm{CV}$ \\
\hline \multirow{2}{*}{ Primera } & RI & 33,14 & $\mathrm{~A}$ & \multirow{2}{*}{ NS } & \multirow{2}{*}{$33 \%$} \\
\hline & $\mathrm{RC}$ & 28,96 & A & & \\
\hline \multirow{2}{*}{ Segunda } & RI & 25,49 & A & \multirow{2}{*}{ NS } & \multirow{2}{*}{$18 \%$} \\
\hline & $\mathrm{RC}$ & 24,19 & A & & \\
\hline
\end{tabular}

\section{Eficiencia del uso de agua}

La cantidad de agua para ambos tratamientos ha sido la misma, ya que en el experimento se ha considerado la aplicación del $100 \%$ de la lámina de riego para ambos tratamientos. Sin embargo la eficiencia del uso de agua en 
el tratamiento del RI ha sido mayor respecto al RC ya que se consiguió un mayor rendimiento en el tratamiento RI.

La eficiencia del uso de agua, se expresa como la producción de camote en Kilogramos por metro cubico de agua aplicado al cultivo, siendo este valor menor en el tratamiento RC cuando se aplica la misma lámina de riego al tratamiento RI.

Tabla 10. Comparación de la eficiencia del uso de agua

\begin{tabular}{lrr}
\hline Tratamientos & \multicolumn{1}{c}{ RC } & \multicolumn{1}{c}{ RI } \\
\hline Rendimiento de RR comerciales $(\mathrm{Kg}$ & 53151,54 & 58629,49 \\
$\left.\mathrm{Ha}^{-1}\right)$ & 3040,45 & 3040,45 \\
Cantidad de agua $\left(\mathrm{m}^{3} / \mathrm{Ha}\right)$ & 17,48 & 19,28 \\
\hline EUA $\left(\mathrm{Kg} / \mathrm{m}^{3}\right)$ &
\end{tabular}

De acuerdo a la información que se muestra en la Tabla 10, se puede indicar que la eficiencia de agua fue aprovechada en un $10,3 \%$ en aquellas plantas expuestas bajo el tratamiento del riego por goteo intermitente en relación al tratamiento del riego por goteo convencional, al aplicar la misma lámina de riego.

\section{Conclusiones}

La cantidad de agua que se empleó para el experimento fue de $3040,45 \mathrm{~m}^{3} \mathrm{Ha}^{-1}$. Este valor representa el $60 \%$ de agua empleada por los productores de camote que usan el riego por gravedad. Si bien es cierto que las cantidades de agua para ambos tratamientos fue la misma al obtener el valor de la eficiencia en el uso del agua los resultados han reportado que se ha obtenido que un 10,3\% fue aprovechado por el tratamiento del RI respecto al tratamiento del RC.

En el tratamiento de riego por goteo intermitente se tienen valores del porcentaje de humedad en el suelo $6 \%$ mayores respecto al tratamiento de riego por goteo convencional. La humedad disponible fue del 11,89\%, mientras que la humedad disponible usada por el cultivo fue de $3,67 \%$ valor que equivale al $30 \%$ de la humedad disponible.

El mayor rendimiento fue observado en el tratamiento RI el cual corresponde el 62,75 $\mathrm{Tn} \mathrm{ha}^{-1}$ frente al 57,16 Tn ha $^{-1}$ teniendo una diferencia de $9 \%$. Sin embargo no se reportaron diferencias significativas para ambos tratamientos.

\section{Literatura citada}

Chamba, L. s.f.. Cultivo del camote para el merdado. Recuperado de: https://es.slideshare.net/ jesusdavidramoscruz/cultivo-delcamote

Garcia, I. y Briones, G. 2007. Sistema de riego por aspersión y goteo. Ediciones Trillas. $2^{\circ}$ Edición, México, $182 \mathrm{p}$.

Goyas, H., A.; Achata, H.; Fano et al. 1990. El camote (batata) en el sistema alimentario del Perú. El caso del valle de Cañete. Sistemas Alimentarios. Serie de investigación $\mathrm{N}^{\circ}$ 5. INIA-CIP. Lima, Perú, 51 p. Recuperado de: http://www.pyrargentina.com.ar/ images/galerias/61-201104281637281.pdf

Jaramillo, 2002. Introducción a la ciencia del suelo. Recuperado de: http://www.bdigital.unal.edu. co/2242/1/70060838.2002.pdf

Morabito, J. y Romay, C. 2001. Evaluación de los sistemas de riego por superficie técnica de caudal discontinuo. Recuperado de: http://www.pyrargentina.com.ar/ images/galerias/61-201104281637281.pdf

Pizarro, F. 1996. Riegos localizados de alta frecuencia (RLAF) goteo, microaspersión, exudación. Ediciones Mundi - Prensa. Madrid, España, 510 p. 